\title{
Neuropatía por compresión de la rama cutánea palmar del nervio mediano
}

\author{
L. Aguilella ${ }^{(1)}$, C. Navarro ${ }^{(2)}$ \\ (1) Unidad de Cirugía de la Mano y Extremidad Superior. Hospital Universitario de la Ribera. \\ ALZIRA. VALENCIA \\ (2) Mutua Asepeyo. VALENCIA
}

Correspondencia:

Dr. Luis Aguilella Fernández

Carretera de Corbera, Km. 1

46600 Alzira (Valencia)

Teléfono: 962458100

Fax: 962458156

e-mail: luis.aguilella@comv.es

\begin{abstract}
Presentamos un caso clínico de neuropatía por compresión de la rama cutánea palmar del nervio mediano, producido por una anomalía en la inserción del tendón del músculo palmaris longus, que fue tratado quirúrgicamente, obteniendo una completa desaparición de los síntomas.
\end{abstract}

Palabras clave: Rama palmar nervio mediano, compresión nerviosa, variaciones anatómicas.
We report a case of entrapment neuropathy of the palmar cutaneous branch of the median nerve, caused by an anomalous tendon of the palmaris longus muscle. It was surgically treated with satisfactory outcome.

Key words: Palmar cutaneous branch median nerve, entrapment neuropathy, anatomical variations.

\section{INTRODUCCIÓN}

$\mathrm{L}$

a rama cutánea palmar $(\mathrm{RCP})$ del nervio mediano es una estructura bien conocida compresión quirúrgica del túnel del carpo. Sin embargo, la neuropatía compresiva de la RCP es una lesión infrecuente, que cuenta con escasas descripciones en la literatura. No obstante, su diagnóstico es sencillo conociendo el trayecto anatómico de la RCP y el área sensitiva que le corresponde. Presentamos un caso de compresión aislada de la RCP, provocada por una anomalía del tendón del músculo palmaris longus (PL), que fue tratado quirúrgicamente con resultado satisfactorio.

\section{CASO CLÍNICO}

Paciente varón de 46 años, diestro, trabajador manual en una factoría de automóviles, que presenta una sintomatología de dolor, parestesias y disestesia en la palma de la mano derecha, con más de tres meses de evolución, que se agrava al realizar esfuerzos. El paciente aporta un estudio neurofisiológico que no muestra alteraciones de los parámetros estudiados. En la exploración física se observa una distribución del área de disestesia que coincide característicamente con el territorio habitualmente inervado por la RCP, sugiriendo su afectación. Mediante un bloqueo anestésico selectivo de la RCP a nivel de la muñeca, se obtiene desaparición inmediata de 


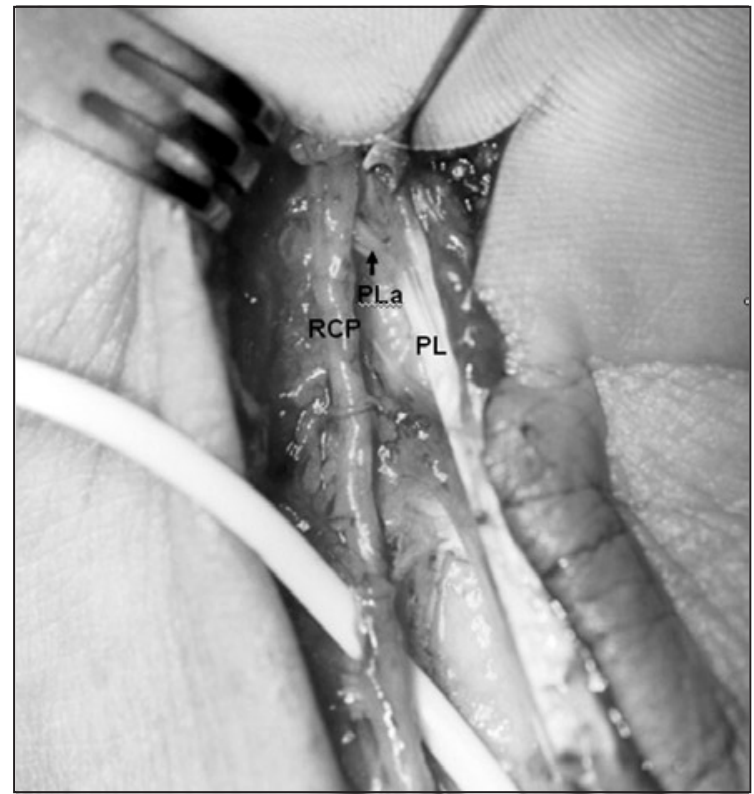

Figura 1. RCP del nervio mediano pasando por el hiato formado entre el tendón del PL y el tendón accesorio (PLa), que es dorsal al RCP.

la sintomatología, confirmándose la sospecha diagnóstica.

El paciente es tratado inicialmente mediante la utilización de una muñequera rígida, que tolera mal en su trabajo diario. También se le practican dos infiltraciones con corticoides,

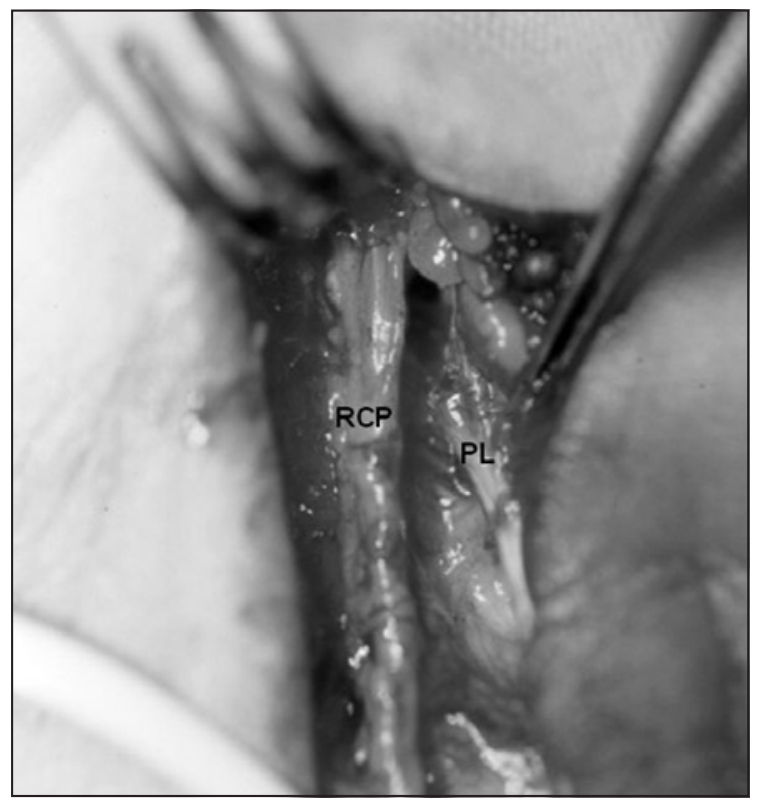

Figura 2. Aspecto de la RCP tras la descompresión por sección de tendón accesorio del PL. obteniendo una mejoría clínica insuficiente y de corta duración. Ante la persistencia de la sintomatología, se decide efectuar tratamiento quirúrgico. En el estudio previo a la cirugía, no se aprecia ninguna anomalía estructural en la radiología convencional ni en la RM de la muñeca. Durante la exploración quirúrgica se observa una zona de compresión de la RCP, ocasionada por la existencia de una bandeleta accesoria del tendón del PL. Este tendón presenta en el paciente una configuración bífida, pasando la RCP por el hiato formado por el tendón del PL y la bandeleta accesoria, que ofrece un aspecto aplanado en la zona de intersección (Figura 1). La simple sección de la bandeleta accesoria, que es dorsal a la RCP (Figura 2), resuelve de forma completa la sintomatología. Dos años después de la cirugía el paciente se mantiene libre de molestias.

\section{DISCUSIÓN}

La neuropatía por compresión de la RCP tiene una sintomatología característica que permite diferenciarla claramente del síndrome del túnel carpiano. El dolor y las parestesias se localizan en la zona de la eminencia tenar, quedando preservada la sensibilidad de los dedos.

La compresión aislada de la RCP del nervio mediano ha sido relacionada principalmente con la presencia de gangliones, que pueden localizarse en cualquier punto del bien descrito trayecto anatómico de la $\mathrm{RCP}^{1-3}$. Otras causas menos frecuentes son la fascia antebraquial o anomalías del músculo PL. También ha sido descrita la sintomatología de disestesias en el territorio de la $\mathrm{RCP}$ en relación con la tendinopatía del flexor carpi radialis ${ }^{4}$.

En el presente caso, la compresión se localizaba en la zona más distal del trayecto de la $\mathrm{RCP}$, al cruzar el tendón del PL, que tenía aquí una forma bífida. Se trataría de un caso en cierto modo similar al presentado por Duncan et al. ${ }^{5}$, en el que coexistía un síndrome del túnel carpiano con la compresión de la RCP al cruzar el tendón accesorio del PL. Dowdy et al. ${ }^{6}$ efectuaron un estudio anatómico sobre 52 muñecas de cadáver, con 49 PL presentes, y encontró dos casos en los que la RCP atravesaba la inserción del tendón 
del PL. Si tomamos en consideración estos datos, no deja de sorprender la escasa frecuencia con la que se diagnostica la neuropatía compresiva de la RCP.
Salvo en algún caso tratado tardíamente ${ }^{7}$, la descompresión quirúrgica resuelve por completo los síntomas de la neuropatía compresiva de la RCP del mediano y se recomienda no diferirlo.

\section{BIBLIOGRAFÍA}

1. Naff $N$, Dellon AL, Mackinnon SE. The anatomical course of the palmar cutaneous branch of the median nerve, including a description of its own unique tunnel. J Hand Surg Br, 1993; 18: 316-7.

2. Al-Qattan MM. Anatomical classification of sites of compression of the palmar cutaneous branch of the median nerve. J Hand Surg Br, 1997; 22: 48-9.
3. Nagle DJ, Santiago KJ. Anomalous palmar cutaneous branch of the median nerve in the distal forearm: case report. J Hand Surg Am, 2008; 33: 1329-30.

4. Gazarian A, Foucher G. La tendinite du grand palmaire. A propos de vingt-quatre cas. Ann Chir Main 1992; 11: 14-18.

5. Duncan GJ, Yospur G, GómezGarcía $A$, et al. Entrapment of the palmar cutaneous branch of the median nerve by a normal palmaris longus tendon. Ann Plast Surg, 1995; 35: 534-6.

6. Dowdy PA, Richards RS, McFarlane RM. The palmar cutaneous branch of the median nerve and the palmaris longus tendon: a cadaveric study. J Hand Surg Am, 1994; 19: 199-202.

7. Gessini L, Jandolo B, Pietrangeli $A$, et al. Compression of the palmar cutaneous nerve by ganglions of the wrist. J Neurosurg Sci, 1983; 27: 241-3. 